\title{
Experiencia en la implementación de sistemas de gestión de calidad para la atención odontológica en un centro docente asistencial universitario.
}

\section{Experience in the implementation of quality management systems for dental care in a university teaching center.}

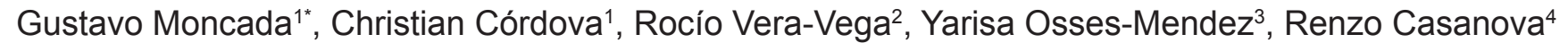

1. Rehabilitación Oral, Facultad de Odontología, Universidad de Los Andes, Chile.

2. Enfermera Calidad Odontológica, Facultad de

Odontología, Universidad de Los Andes, Chile.

3. Enfermera Consultas Médicas, Universidad de

Los Andes, Chile

4. Director Odontológico Centro de Salud San

Bernardo. Facultad de Odontología, Universidad de Los Andes, Chile

* Correspondencia Autor: Gustavo Moncada | Dirección: Mons. Álvaro del Portillo 12.455, Las Condes, Santiago, Chile. | E-mail: gmoncada@ adsl.tie.cl

Trabajo recibido el 08/04/2020.

Trabajo revisado 13/07/2020

Aprobado para su publicación el 15/08/2020

\begin{abstract}
RESUMEN
Objetivo: Describir el desarrollo e implementación para el establecimiento, cumplimiento y acreditación como centro de atención abierta, para la atención odontológica de un centro docente asistencial. Metodología: Para alcanzar mayores niveles de calidad sanitaria y lograr satisfacción de pacientes, profesionales, estudiantes, personal auxiliar y administrativo, definimos 8 niveles de acción: orientar trabajo a resultados; realizar actividades centradas en el paciente; desarrollar el liderazgo y coherencia en los objetivos; gestionar por medio de procesos; involucrar a toda la comunidad; incorporar a la gestión el aprendizaje, la innovación y la mejora constante; potenciar alianzas internas y externas y garantizar la responsabilidad social. Resultados: El comité de calidad rediseñó protocolos, realizó mejoras computacionales y administrativas, realizó diálogos con la comunidad, incrementando de $80 \%$ a $100 \%$ el cumplimiento de los estándares obligatorios $(n=11)$ y de $70 \%$ a $96 \%$ los globales. Conclusiones: Alcanzar las competencias de planificación, gestión sanitaria y elevar los niveles de calidad en la atención de pacientes de un centro de salud que incluye una Facultad de Odontología, es un desafío continuo en el tiempo que involucra toda la comunidad, requiere especialistas del área, incrementa los costos de operación y conduce a su acreditación como centro de atención abierta.
\end{abstract}

\section{PALABRAS CLAVE}

Calidad en atención del paciente; Educación odontológica; Acreditación en calidad; Centro de atención abierta.

Int. J. Inter. Dent Vol. 14(1); 11-16, 2021.

\section{ABSTRACT}

Objective:To describe the development and implementation for the establishment, fulfillment and accreditation as an open-care center, for dental care in a University Teaching Center. Methodology: In order to reach higher levels of health care quality and additionally achieve the satisfaction of patients, professionals, students, auxiliary and administrative staff, the quality committee developed 8 levels of action: 1, orienting the work to results; 2 , performing patient-centered activities; 3 , developing leadership and coherence in the objectives; 4 , managing through processes; 5 , involving the entire community; 6 , incorporating learning, innovation and constant improvement into management; 7 , strengthening internal and external alliances and 8 , ensuring social responsibility. Results: After 4 years of work by the quality and review committees, with the redesign of protocols, computer and administrative improvements, evaluation of progress and dialogue with the community, compliance with mandatory quality standards $(n=11)$ was increased from $80 \%$ to $100 \%$ and compliance with the global standard was increased from $70 \%$ to $96 \%$ (mandatory + non-mandatory standards). Conclusions: The strategy to achieve planning and management competences in the health system and to raise quality levels of patient care in a health center that includes a dental school is a continuous challenge over time that involves the entire community, requires specialists in the area, increases operating costs and leads to accreditation as an Open.Care Center.

\section{KEY WORDS}

Quality patient care; Dental education; Quality accreditation; Open center of attention.

Int. J. Inter. Dent Vol. 14(1); 11-16, 2021. 


\section{INTRODUCCIÓN}

La atención sanitaria de calidad involucra la identificación completa y precisa, de las necesidades de salud de los individuos o de la población, destinando oportunamente los recursos para satisfacer estas necesidades, siendo tan efectiva como el estado actual del conocimiento lo permita. En la atención sanitaria de calidad confluyen cuatro elementos esenciales: A, alto nivel científico-técnico; B, seguridad (menor riesgo posible); C, eficiencia (costos mínimos de recursos) y $\mathrm{D}$, máximo nivel de satisfacción del paciente ${ }^{(1-5)}$.

El interés sobre la calidad de la atención en salud ha aumentado su representación durante los últimos años, incluida la odontología y la educación dental. Al considerar que la medición es un elemento clave para mejorar la calidad, se han desarrollado encuentros de las principales partes interesadas en el cuidado de la salud oral para liderar el desarrollo de medidas y determinación de su rendimiento. Es relevante evaluar el desempeño de programas y prácticas en el ejercicio de la odontología. Estas medidas complementan los niveles de calidad que aplican las escuelas de odontología, son utilizadas para garantizar el cumplimiento de las normas y así respaldar los procesos de acreditación de la calidad de las prestaciones ${ }^{(6,7)}$

En Estados Unidos (EE.UU.), el Foro Nacional de la Calidad (NQF) definió las medidas de calidad como las herramientas utilizadas para cuantificar la atención que se proporciona a los pacientes y evaluar cómo mejorar la atención o los resultados, en diferentes condiciones y durante un período de tiempo específico ${ }^{(8)}$.

El interés por la calidad de la atención odontológica tradicionalmente ha estado centrada en lo clínico procedural y su desarrollo integral ha sido más lento que en el área médica. Los cuidados por la calidad en salud han ganado adeptos que se incrementan durante la última década y lentamente están logrando su espacio en la profesión odontológica ${ }^{(6)}$.

El beneficio del establecimiento de protocolos clínicos y de la constante preocupación por su actualización conduce a mejoras en la calidad de la atención, como recientemente lo demuestra la revisión periódica de la evidencia y protocolos para el manejo clínico de la enfermedad caries, que acoge el actual cambio de paradigma científico, ha alcanzando importantes modificaciones sanitarias al observar cambios en el modelo de educación odontológica, donde la revisión del currículo motivado por la acreditación, condujo en Australia, a la migración de un modelo restaurador a un modelo no quirúrgico para el manejo de la caries dental en etapas precoces, demostrando la reducción del 30 al 50\% de las intervenciones restauradoras después de 7 años ${ }^{(9)}$. Otro ejemplo de detección de problemas en la atención odontológica de calidad, lo muestra el análisis sobre certificación y desarrollo de los implantes dentales para los próximos 10 años, concluyendo que la ingeniería dental, las enfermedades peri-implantarias y el flujo de trabajo digital en odontología actualmente tienen herramientas limitadas que no garantizan resultados clínicamente predecibles ${ }^{(10)}$.

El modelo de acreditación destinado a promover la mejora e innovación en la atención sanitaria, se alcanza con el compromiso de las máximas autoridades de los servicios y en este caso de la Universidad, mejorando directamente la cultura de calidad de la organización, existiendo compromiso con los pacientes, el entorno y la sociedad, detectando insuficiencias y fortalezas de la organización y especialmente respetando la cultura organizacional, hechos que determinan las pautas para la mejora de los procesos, con respeto por el medio ambiente ${ }^{(21,22)}$.

La atención sanitaria de calidad se sustenta en un conjunto de normas legales, desplegadas por el Gobierno de Chile, entre las que destacan la definición de los procesos de atención sanitaria (según normativa vigente a la fecha de la acreditación) del Ministerio de Salud (MINSAL, Decreto Fuerza de Ley $\left.\mathrm{N}^{\circ} 1,2005\right)^{(11)}$, procesos que se aplican y detallan su ejecución según el Reglamento del Sistema de Acreditación para los Prestadores Institucionales de Salud (D. S. N ${ }^{\circ}$ $15 / 2007$, MINSAL) $)^{(12)}$ y adicionalmente establece los estándares mínimos y contenidos normativos que son detallados en el Manual del Estándar General de Acreditación para Prestadores Institucionales de Atención Abierta(13) $^{(13)}$ donde se desagregan las exigencias separadas por ámbitos, componentes, características e indicadores.

Los prestadores institucionales autorizados por la Autoridad Sanitaria, tales como: hospitales, clínicas, centros ambulatorios y laboratorios se acreditan en un proceso periódico de evaluación externa, con el objetivo de verificar el cumplimiento del conjunto de estándares de calidad fijados y normados por el Ministerio de Salud. Dicho proceso de evaluación es ejecutado por Entidades Acreditadoras que son autorizadas por la Superintendencia de Salud, inscritas en el Registro Público de Entidades Acreditadoras $^{(14)}$.

La acreditación de prestadores institucionales es voluntaria y se considera el inicio de la estrategia destinada a alcanzar competencias de planificación y gestión en el sistema sanitario, estableciendo programas, orientados a promover la filosofía de mejora continua de la calidad en los centros de salud, sin embargo, para la vigencia de la Garantía Explícita de Calidad de las prestaciones garantizadas por GES/AUGE, el prestador interesado en otorgar estas prestaciones debe estar obligatoriamente acreditado para otorgar la prestación garantizada ${ }^{(15)}$. Las actuales normas fueron diseñadas básicamente para ambientes de atención médica no educacional, sin embargo, sus características generales son claramente aplicables a la gestión clínica odontológica en un centro educacional.

El establecimiento y cumplimiento de los estándares de calidad para la atención odontológica incluye todo lo que realiza el equipo de salud para la atención de los usuarios y estos procedimientos se certifican por medio del sistema de acreditación, que incluye nueve ámbitos evaluables durante el proceso de acreditación; Respeto a la dignidad del paciente (DP), Gestión de la calidad (CAL), Gestión clínica (GLC), Acceso, oportunidad y continuidad de la atención (AOC), Competencias del Recurso Humano (RH), Registros clínicos (REG), Seguridad del equipamiento (EQ), Seguridad de las instalaciones (INS) y Servicios de apoyo: Servicio de Esterilización (APE), Servicio de Imagenología (API), Servicio de Kinesiología (APK) y Servicio de Farmacia (APF) ${ }^{(16)}$.

El objetivo del presente artículo es describir el desarrollo e implementación para el establecimiento, cumplimiento y acreditación como Centro de Atención Abierta, para la atención odontológica en un Centro Docente Asistencial Universitario.

\section{METODOLOGÍA}

Cronología de las actividades: La autoridad universitaria define la necesidad de implementar el establecimiento y cumplimiento de estándares de Centro de Atención Abierta (CAA), para la atención odontológica en el Centro Docente-Asistencial de San Bernardo (CESA), (2012). La dinámica administrativa y funcional de las instituciones en San Bernardo son la consecuencia de definiciones institucionales que delimitan al CESA como una Unidad de Apoyo Académico, perteneciente a la Universidad de Los Andes, que presta servicios docente-asistencial a las facultades de Medicina y Odontología y a las escuelas de Enfermería, Obstetricia, Psicología, Terapia Ocupacional y Fonoaudiología. La U. de Los Andes cede parte de la infraestructura del CESA al Hospital Parroquial de San Bernardo (HPSB), entidad administrada por una Fundación del Arzobispado de Santiago, para la realización de consultas médicas ambulatorias externas, a beneficiarios Fonasa del Servicio de Salud Metropolitano Sur. Adicionalmente, el HPSB funciona como campus clínico de algunas especialidades médicas y de la rotación de los alumnos de último año de la Facultad de Odontología. EI HPSB le brinda servicios al CESA, como entidad externa, en las áreas de Laboratorio de Medicina Patológica, como parte de la actividad docente asistencial, cumpliendo con los requisitos y estándares de trazabilidad de muestra, junto con servicios de exámenes de laboratorio clínico, como el test rápido para $\mathrm{VIH}$ de la muestra del paciente foco, como parte del protocolo de manejo de accidentes cortopunzantes.

La cultura de calidad en el CESA, se inicia formalmente el año 2013, desarrollando protocolos, definiendo pautas de supervisión y verificadores. El año 2014, se conforman los Comités de Calidad, Ética y Farmacia. El 2015 hasta la fecha, se perfeccionan constantemente los documentos, protocolos, pautas, verificadores, actas y certificados de los comités.

Durante los meses de enero y febrero de 2016 se contratan los servicios de una enfermera universitaria experta en calidad, quién realizó sus mayores aportes en la ficha clínica única y los registros profesionales en la Superintendencia de Salud de los alumnos de las especialidades odontológicas.

El 30 de mayo de 2016 la empresa acreditadora, Acredita Norte Chile Ltda. inicia la evaluación del CESA y concluye que el prestador cumple con el $80 \%$ de las características obligatorias y el $70 \%$ de la características globales que se les aplican. Dado que para el primer proceso de acreditación se exigía el cumplimiento del 100\% de las características obligatorias aplicables y el cumplimiento del $50 \%$ de las características totales, el CESA no acredita. Las características incumplidas fueron CAL 1.1 (falta el análisis de la programación del programa de calidad) y en REG 1.1 (RUT como único número identificador de pacientes).

Las acciones previas a la segunda acreditación consideraron tres niveles de acción, el trabajo interno destinado a consensuar prácticas de calidad y sociabilizar información con la comunidad sanitaria del CESA; analizar e implementar lo informado por una empresa de Asesoría en Calidad y Acreditación Limitada (ACA) y el abordaje de las tareas definidas en los antecedentes de la experiencia de acreditación no alcanzada el año 2016, que generó un conjunto de medidas que se adoptaron a contar del año 2017.

Para liderar el trabajo interno se constituyó el Comité de Calidad 
(CC) formado por los 13 jefes de los servicios del CESA (Gerente, Director médico, Director Clínico Odontológico, Director de Salud Mental, Enfermera Universitaria (EU) de Calidad, EU de Odontología, EU de pabellón, EU de Infecciones Asociadas a Atenciones de Salud (IAAS), EU Consultas Médicas, Coordinadora de Salud Mental, encargada de atención al paciente, Jefe Administrativo y Jefe de personas). Adicionalmente se constituyó el Comité Revisor, encargado de la supervisión de los protocolos y documentos de trabajo constituido por el jefe del área correspondiente, la EU de calidad y en casos de ausencia de alguno de ellos, es reemplazado por el Director médico y/o el Gerente del CESA.

El CC consideró la inclusión de profesores, alumnos y funcionarios como el eje central de los procesos para generar la cultura institucional, conducente al desarrollo y perfeccionamiento de los protocolos de atención al paciente y a la acreditación como CAA.

La primera etapa que permitió conocer las debilidades y fortalezas del Centro, se basó en definir la calidad como condición esencial del sistema sanitario y las acciones creadas para su mejora continua intentaron incluir todas las facetas de los procesos asistenciales, iniciado con la difusión de la preocupación por la calidad, la incorporación activa de profesionales, funcionarios y estudiantes, la promoción de las mejoras percibidas por los pacientes expresadas en el trato y la información recibida. Centro de este proceso fue enfocar la tarea en el conocimiento, entendimiento y aceptación desde el punto de vista del paciente y secundario, pero no menos importante, el acatar normas y estándares científico técnicos de la profesión ${ }^{(19)}$, especialmente cuando las percepciones de los pacientes superan la de los funcionarios de la salud en Chile ${ }^{(20)}$.

Como instrumento pauta para informaciones, requisitos y etapas del proceso de construcción y cumplimiento de las garantías de calidad a seguir, el CC utilizó la Guía Práctica para el Proceso de Acreditación de Prestadores Institucionales de Salud(17).

El impacto de las actividades realizadas por los profesionales, alumnos y funcionarios, fue medido por autoevaluaciones internas, lideradas por la EU de calidad del CC, quién veló por el cumplimiento de las rúbricas de evaluación como CAA y verificó el cumplimiento de los estándares establecidos.

Para alcanzar mayores niveles de calidad sanitaria y adicionalmente, lograr satisfacción de los pacientes, profesionales, estudiantes, personal auxiliar y administrativos, el comité fundamentó sus tareas basado en los siguientes ocho niveles de acción (descritos como objetivos en los resultados): 1, Orientar el trabajo a los resultados; 2, Orientar las actividades del Centro de Salud en el paciente; 3, Desarrollar el liderazgo y la coherencia en los objetivos; 4, Gestionar por medio de procesos; 5 , Desarrollar e involucrar a todos los profesionales, estudiantes y funcionarios; 6, Incorporar a la gestión el aprendizaje, la innovación y la mejora. 7. Desarrollar y potenciar las alianzas internas y externas y 8 , Garantizar la responsabilidad social.

La cuantificación del cumplimiento de las características que aplican al prestador, se efectuó utilizando la pauta de cotejo del estándar general, para prestadores institucionales de atención abierta del MINSAL, que define los porcentajes y áreas de cumplimiento(17,18).

\section{RESULTADOS}

Los resultados se presentan clasificados según la propuesta de los 8 niveles de acción institucional, donde se acogen los nueve ámbitos evaluables durante el proceso de acreditación.

El objetivo o nivel de acción 1, de orientar el trabajo a los resultados, se implementó parcialmente, dado que como estrategia de gestión no se permitió a las personas desempeñarse por resultados en vez de horas trabajadas, pues la clínica requiere horario definido de funcionamiento. Sin embargo se implementó en el sentido de actuar con velocidad y con sentido de urgencia en la solución de los problemas integrando los estándares requeridos.

En el primer proceso, (2016, resultado en no acreditación), se cumplió el $80 \%$ ( 8 de 10 ) de los estándares obligatorios y el $62,5 \%$ ( 25 de 40 ) de los no obligatorios. Determinando el cumplimiento global del $70 \%$ de los estándares aplicables al prestador (35 de 50).

Los incumplimientos de los estándares obligatorios en la primera acreditación correspondieron a Gestión de calidad (CAL1.1) y Registros Clínicos (REG 1.1). Para CAL 1.1 se identificó como carencia en el informe anual del análisis de los resultados, la respuesta fue establecimiento del análisis de los resultados del informe anual a contar del año 2017 y desarrollo de un programa de trabajo que estableció el sistema de evaluación de calidad, definiendo responsables, plazos para la evaluación anual y frecuencia de actualización de la política de calidad cada tres años.

En registros clínicos (REG1.1), se agregaron los antecedentes de enfermería de pabellón a la ficha clínica única (FCU), quedando definido el acceso estandarizado a la FCU solo por medio del RUT, manteniéndose el acceso por número de pasaporte para extranjeros sin RUT, instruyéndose al departamento de tecnologías de la información (DTI) el diseño de un sistema que evite fichas duplicadas de pacientes en el programa Salud. En el standard REG 1.1 se resolvió la dificultad para acceder a la información de la atención odontológica efectuada (a un paciente seleccionado al azar), en su ficha electrónica y en el sistema de imágenes radiográficas (SIDEXIS), por medio del re entrenamiento de todos los miembros de la comunidad y renovación de la totalidad de los equipos digitales incrementando sus capacidades operativas, hecho que permitió el acceso a la versión actualizada de todos los registros clínicos y documentos, desde todos los puntos de trabajo institucional, mejorando la característica REG1.2, además se definió la frecuencia de renovación de equipamiento de acuerdo a la obsolescencia de los equipos.

Para el estándar AOC2.1 (acceso, oportunidad y continuidad de la atención), que posee umbral del $100 \%$ y se cumple tanto el año 2016 como el 2019, se decidió incorporar un documento de información para él paciente, que facilitó la información y seguimiento cuando es derivado a otras especialidades. Este documento de información acompaña físicamente al paciente, además de la orden digital interna a contar del año 2017.

2. En el nivel de acción orientar las actividades del Centro de Salud en el paciente se perfeccionó el standard dignidad del paciente (DP1.2) procediendo a actualizar el texto del documento de reclamos para acoger y responder a los reclamos y/o sugerencias de los pacientes dentro de 48 horas. Para la evaluación de los derechos de los pacientes (DP1.3) se estableció un profesional centralizado responsable y evaluador del documento de reclamos y/o sugerencias, se determinó la forma de tabular los datos, responder los reclamos y su seguimiento, concluyendo con la confección de la estadística anual de los indicadores. Adicionalmente se incorporó la encuesta de satisfacción de la atención al paciente y la forma en que procesa y tabula los datos, el mismo profesional responsable.

La dignidad del paciente es el eje de acción del Centro, implementándose mecanismos de información del diagnóstico, pronóstico y plan de tratamiento odontológico integrado a cada paciente y a cada intervención, promoviendo la atención de un paciente informado y consiente de sus derechos, los que se publicitan en los muros del centro (8 gigantografías). Se actualizaron todos los convenios docente asistencial y se determinó el responsable de los convenios y la frecuencia de su revisión (DP4.1).

Se definieron las situaciones en que deben someterse a evaluación ética y el procedimiento para la presentación de casos al Comité, se estandarizan y actualizan los documentos del Comité de Ética Asistencial y se procedió a designar sus funciones y responsables, periodicidad de sesiones, modalidad de registros y firma de los escritos y confirmación de sus miembros, además de informar a la comunidad (DP5.1)

3. Para desarrollar el liderazgo y la coherencia en los objetivos se consideró como eje central el recurso humano, promoviendo a los líderes naturales de las diferentes áreas de trabajo, con el objetivo de potenciarlos como modelos de los procesos y principalmente éticos, hecho que permitió ensamblar los objetivos estratégicos del CC con cada miembro o colaborador de la comunidad, situación que fue más allá de la simple designación, transformándose en cultura de valoración y creación de empoderamiento para el logro de las metas del CESA.

4. En gestión por medio de procesos, se organizó el trabajo en función del mejoramiento continuo de las actividades dentro del centro, inicialmente se realizó como principal esfuerzo el asegurar la trazabilidad del proceso de biopsias, desde la toma de muestras hasta la información del resultado al paciente. Todas las biopsias o muestras citológicas tomadas en el centro se registran en el "libro de trazabilidad" según corresponda al servicio. En el caso del servicio dental, el cirujano dentista que toma la biopsia, completa la solicitud, avisa a la enfermera. Se almacema la muestra en un frasco ad hoc y se rotula. Luego se realiza el registro en el libro de trazabilidad biopsias en donde se reporta nombre y apellidos del cirujano dentista quien se adjudica el rol de custodio, luego el personal técnico de enfermería acude para trasladar la muestra al Hospital Parroquial de San Bernardo (registrado como custodio 2 en el libro) y para su control el libro de trazabilidad es revisado diariamente por la enfermera encargada.

Una vez que se reciben los resultados se adjuntan a la ficha del paciente y se informa via mail a la enfermera y al director odontológico. En caso de resultados alterados, se activa el protocolo de notificación de resultados críticos, informando al paciente via telefónica o mediante carta certificada en caso de ser necesario. También se notifica al médico o dentista tratante, luego se realiza el registro en el "libro de notificación de resultados críticos".

En seguridad del equipamiento, fue otro proceso donde se detectaron 
deficiencias, como el caso del mantenimiento de las autoclaves, generando el libro foliado de respaldo del mantenimiento, se designó su responsable y definieron los criterios de control y evaluación anual (GLC 1.8).

5. Para el objetivo de desarrollar e involucrar a todos los profesionales, estudiantes y funcionarios, se trabajó la promoción de ambientes motivados, productivos e innovadores, realizando reuniones informativas, junto con la sociabilización de protocolos, segmentadas por estamento. También se diseñó, imprimió y distribuyó a todo los miembros de la comunidad, un manual de bolsillo, con información detallada de los principales aspectos de la cultura de calidad en el CESA, que contenía protocolos, responsables por área, fonos de emergencia y horarios de funcionamiento (AOC 1.1).

6. La incorporación de la gestión del aprendizaje, logró reflejar en el quehacer educacional la responsabilidad de formar personas, promoviendo el auto-perfeccionamiento, la innovación y la mejora constante de los procesos, teniendo como meta la promoción con especial énfasis, en el análisis y tratamiento de los resultados de los informes anuales a contar del año 2017 (AOC 1.2).

7. Como consecuencia de todas las metas propuestas anteriormente se logró desarrollar y potenciar las alianzas internas, comunicación entre los diferentes servicios internos y externos del CESA con el Hospital Parroquial donde se inició el proceso e informe de las biopsias y recepción y tratamiento de los accidentes corto punzantes (GCL 2.2).

8. Para desarrollar el objetivo de garantizar la responsabilidad social el CC propuso y concretó prácticas, estrategias y sistemas destinados a mejorar la autoayuda, apoyo a la comunidad como contribución activa y voluntaria al mejoramiento social y ambiental de las personas (DP 5.1).

En el proceso de diseño de cada protocolo se identificó el nombre de la medida o ámbito de acción, código o número e incluyo los objetivos, alcances, definiciones operacionales, materiales y equipos, responsables, equipo o personal operativo y encargado de la calidad, procedimientos y diagramas explicativos, flujo de notificaciones o algoritmo de aplicación u operación, indicadores de cumplimiento expresados en porcentajes y anexos como formularios, pautas de supervisión, solicitudes, ordenes de atención u otros, según el caso.

Los protocolos de mayor impacto fueron; Protocolo de vigilancia de eventos adversos (EA) y centinelas (EC) (GCL 2.2). Normativa de Notificación GES Clinica Odontológica centro de salud de la Universidad de Los Andes. Protocolo acceso ficha electrónica. Protocolo acceso a imágenes. Protocolo Código azul (AOC 1.1) y plan de evacuación. Protocolo para manejo emergencias médicas. Protocolo para accidente corto punzante. Protocolo acceso a comité de ética. Protocolo acceso a ficha clínica electrónica. Protocolo de adquisición de imágenes (API 1.3). Requisitos de solicitudes e indicaciones generales de exámenes imagenológicos y resumen de protocolos

Históricamente, la Facultad de Odontología ha sido el servicio que atiende la mayor cantidad de pacientes del Centro (Tabla 1).

Tabla 1: Distribución de la cantidad de pacientes atendidos durante el período 2015-2018 por los diferentes servicios de salud del CESA expresados en cantidad de pacientes y porcentaje del total.

\begin{tabular}{l|c|c}
\hline & Pacientes (N) & $\%$ \\
\hline Odontología & 255.221 & 58.5 \\
\hline Salud Mental & 14.770 & 3.3 \\
\hline Consultas Medicas & 50.713 & 11.7 \\
\hline $\begin{array}{l}\text { Kinesiología, Ecografía, Nutrición, } \\
\text { Pabellones, Fonoaudiología }\end{array}$ & 28.847 & 6.6 \\
\hline Beneficiarios Salud Sur & 85.120 & 20.0 \\
\hline Total & 434.671 & 100.1 \\
\hline
\end{tabular}

Después de 4 años de trabajo del comité de calidad, comité revisor, rediseño de protocolos, implementaciones computacionales y administrativas, evaluación de avances y diálogo con la comunidad, se logró incrementar de $80 \%$ a $100 \%$ el cumplimiento de los estándares de calidad de las 11 características obligatorias y de $70 \%$ a $96 \%$ de los 28 estándares no obligatorios (Gráfico 1 y Tabla 2).

\section{DISCUSIÓN}

La implementación de sistemas de gestión de calidad para la atención odontológica y el proceso de acreditación consideró importantes ventajas
Evaluación de las características obligatorias según evaluación 2016 y 2019.

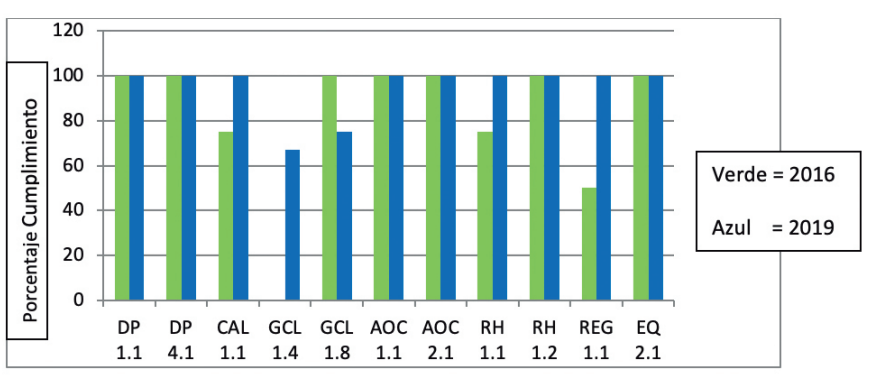

Caracteristicas Obligatorias

Gráfico 1: Resumen de la evaluación de las características obligatorios según evaluación $2016(\mathrm{~N}=10)$ y $2019(\mathrm{~N}=11)$.

Tabla 2: Resumen de la evaluación de calidad de las 28 características no-obligatorios según evaluación 2016 y 2019.

\begin{tabular}{|c|c|c|c|}
\hline \multicolumn{4}{|c|}{ Característica No Obligatorios Evaluadas } \\
\hline Código & $\begin{array}{c}\% \\
\text { Cumplimiento } \\
\text { Agosto } 2016\end{array}$ & $\begin{array}{c}\% \\
\text { Cumplimiento } \\
\text { Abril } 2019\end{array}$ & \multirow{2}{*}{$\begin{array}{c}\begin{array}{c}\% \text { Umbral } \\
\text { Exigible }\end{array} \\
\text { MINSAL }\end{array}$} \\
\hline & \multicolumn{2}{|c|}{ Súper Intendencia de Salud } & \\
\hline DP 1.2 & 100 & 100 & 100 \\
\hline DP 1.3 & 100 & 100 & $\geq 66$ \\
\hline DP 3.1 & 100 & 100 & $\geq 66$ \\
\hline DP 4.2 & 100 & 100 & $\geq 75$ \\
\hline DP 5.1 & 50 & 100 & $\geq 75$ \\
\hline GCL 1.3 & 100 & 100 & $\geq 75$ \\
\hline GCL 1.5 & 100 & 100 & $\geq 75$ \\
\hline GCL 2.2 & 100 & 100 & $\geq 75$ \\
\hline GCL 3.1 & 100 & 100 & 100 \\
\hline GCL 3.2 & 100 & 67 & $\geq 75$ \\
\hline AOC 1.2 & 75 & 100 & $\geq 75$ \\
\hline $\mathrm{RH} 1.3$ & 100 & 100 & $\geq 50$ \\
\hline RH 2.1 & 100 & 100 & 100 \\
\hline RH 2.2 & 40 & 100 & $\geq 75$ \\
\hline RH 3.1 & 33 & 100 & $\geq 50$ \\
\hline RH 4.1 & 75 & 67 & $\geq 75$ \\
\hline $\mathrm{RH} 4.2$ & 71,4 & 100 & $\geq 75$ \\
\hline REG 1.2 & 85,7 & 100 & $\geq 75$ \\
\hline REG 1.4 & 66 & 100 & $\geq 66$ \\
\hline EQ 1.1 & 100 & 100 & 100 \\
\hline EQ 1.2 & 50 & 100 & 100 \\
\hline EQ 2.2 & 50 & 100 & 100 \\
\hline EQ 3.1 & 100 & 100 & 100 \\
\hline INS 1.1 & 60 & 100 & $\geq 80$ \\
\hline INS 2.1 & 72,7 & 100 & $\geq 75$ \\
\hline INS 2.2 & 0 & 100 & $\geq 75$ \\
\hline INS 3.1 & 66 & 100 & 100 \\
\hline INS 3.2 & 25 & 100 & $\geq 75$ \\
\hline
\end{tabular}

en la gestión de calidad para pacientes y profesionales. Fomento interdisciplinaridad, desarrollando un proyecto común entre personal directivo, profesionales, administrativos y estudiantes, adicionalmente incorporó un modelo de gestión participativo, promotor de los cambios y finalmente añadió valores como la coordinación, integración y 
observación continua de los procesos asistenciales.

La experiencia de la no acreditación el año 2016, concluyó que del estándar general, de 90 características contenidas, para prestadores institucionales de atención abierta, correspondía la aplicación de 50 al CESA, siendo 10 estándares de carácter obligatorios y 40 no obligatorios. La norma considera que el estándar mínimo de cumplimiento aplicable al año 2016 (Decreto Exento №18 del MINSAL), es el 100\% de las características obligatorias y del $50 \%$ de las características globales (Obligatorias más no Obligatorias) aplicables al centro a acreditar (Tabla 2).

Cabe destacar la modificación que se hace del decreto exento $\mathrm{N}^{\circ} 18$ de 2009, en relación a flexibilizar las exigencias para lograr la acreditación a partir del 15 de enero de 2019, pudiendose alcanzar una "acreditación con observaciones", para entidades que cumplan con al menos el $50 \%$ de las características que les fueron aplicadas y acepta el incumplimiento máximo de 2 categorías obligatorias. De esta manera no se perjudica a los centros que invierten gran cantidad de recursos para obtener dicha certificación. Se efectúa la revalorización en las caracteristicas obligatorias de alto umbral, ya que existe necesidad en aumento de asegurar la calidad de los servicios sanitarios y de avanzar en la instalación de sistemas de acreditación. Es decir para los estándares actuales habríamos acreditado el CESA del año 2016 con observaciones.

Reglamentariamente la no acreditación no define el tiempo de espera para la próxima acreditación, solo define el cambio de la agencia acreditadora, sin embargo no se volvió a intentar hasta tener certeza de logros que permitieran su acreditación, lo que ocurrió 3 años después. Se sufre el impacto de asumir un desafío no superado, se toma conciencia de la importancia del equipo y sus roles en la acreditación. Sirve para darse cuenta que este desafío debe ser asumido con mucha seriedad, responsabilidad y con lo mejor del recurso humano disponible en el área. La conducta posterior consiste en re organizar el equipo, definir responsabilidades y comenzar a trabajar rápidamente con una agencia asesora.

Del total de 12 características obligatorias aplicables al prestador, el primer informe de la Superintendencia de Salud evaluó 10 (año 2016), mientras el año 2019 evaluó 11, incluyendo el manejo de pacientes con tratamiento anticoagulantes (GCL 1.4), siendo para este estándar el umbral exigido mayor o igual al $66 \%$, logrando el total cumplimiento de los estándares obligatorios, este hecho fue recibido con sorpresa, pero luego se comprendió que estaba dentro de las atribuciones del evaluador.

Durante el proceso, fue tarea constante para el cumplimiento de los estándares, el alinear el respaldo institucional con la organización interna conducida por el Comité de Calidad y el Comité Revisor que permitieron el desarrollo, documentación e implementación del establecimiento y cumplimiento de estándares de calidad para la atención odontológica en el CESA, de acuerdo con los estándares propuestos por el Manual del Estándar General de Acreditación para Prestadores Institucionales de Atención Abierta, del Ministerio de Salud, que fue guía y consulta permanente. Al igual que transmitir a la comunidad el espíritu de la cultura de calidad, el desarrollo de protocolos, definiendo pautas de supervisión y verificadores, actas y certificados de respaldo.

Aspectos formales de la dinámica de calidad se transformaron en vitales, destacando las unidades de gestión que requirieron cada vez mayor dedicación, que los grupos de pequeño tamaño con que se inició la tarea.

La participación de los miembros de la comunidad en los procesos de calidad fue la actividad de mayor desafío, especialmente en el grupo de profesionales que dedican pocas horas al servicio y/o la docencia. El proceso de aprendizaje de académicos, funcionarios y estudiantes presentó diferentes dinámicas, tiempos y resultados, siendo las auxiliares dentales y de botiquín junto con los estudiantes, quienes adhirieron con mayor facilidad a los cambios.

La implementación digital significó un gran apoyo a las labores de calidad, porque estandarizó los procesos, sin embargo la interconexión de los diferentes servicios fue una tarea que no siempre fue adecuadamente prevista, especialmente para mantener en todas las prestaciones de salud del Centro niveles similares de calidad.

En un servicio altamente digitalizado, como camino a institución libre de papel, se requirió extremada precisión en la definición de los procesos de acceso y seguridad digital, que permitió que toda la comunidad trabajara en forma estandarizada en los registro de la información como ficha clínica única, informes, imagenología, derivaciones, interconsultas y todos los procedimientos administrativos, que permitieran el acceso a datos estadísticamente válidos cuando sea requerida, para esta labor, la pre-evaluación efectuada por una agencia asesora externa ayudó a resolver situaciones que internamente no se visualizaban con la profundidad que detectó el análisis externo. La atención de la Clínica Odontológica libre de papel, implementó sus correspondientes protocolos de operación, permaneciendo actualmente pendientes digitalizar el consentimiento informado, acceso a la clínica de los pacientes y entrega de instrumental a los boxes de atención aún en papel.

El proceso experimentado logró que la calidad en la atención de los pacientes sea un componente transversal en la organización, destacando que para el inicio de los procesos de preparación, fue vital la generación de la cultura de calidad en profesionales, funcionarios y alumnos relacionados con los procedimientos que se realizan en la institución y definiendo que todos son participantes activos y necesarios para el logro de los objetivos.

CESA es el primer Centro Docente Asistencial Acreditado que incluye la clínica de una Facultad de Odontología. Al 24 de junio de 2019, de los 627 registros de prestadores acreditados en la Superintendencia de Salud, mayoritariamente eran Hospitales, Clínicas, Centros Médicos, Laboratorios Clínicos, Políclinicos y Servicios Médicos, 140 estaban registrados como Centros de salud, familiar, nefrológico, mutual, médico, de diálisis, oftalmológico y/o radiológico, a esa fecha 24 centros dentales se encontraban acreditados. Destacándose que del total 38 inscripciones se encuentran canceladas ${ }^{(14)}$.

En resumen, el desarrollo de los estándares de un centro universitario asistencial de atención abierta, significó para una Facultad de Odontología la implementación de diferentes medidas en diversos ámbitos, como por ejemplo la introducción de nuevo personal al Centro y su inducción continua a los procesos, desde las declaraciones de la misión, visión hasta protocolos clínicos, actividades que son actualmente programas regulares en los planes de estudio, incluidos el manejo de programas computacionales de imágenes, ficha clínica única y otros, que determinaron la permanente formación de profesionales y alumnos, al igual que el entrenamiento constante en modalidad de interconsultas y derivaciones. Acciones periódicas son el entrenamiento en código azul, accidentes corto punzantes, lavado de manos e instrumental, plan para emergencias naturales por medio de la definición de líderes de evacuación y sus roles (lugares de evacuación, uso de los chalecos amarillos, megáfonos con pilas cargadas, etc). Otro aspecto relevante a considerar fue el soporte computacional que definió el aumento de la capacidad de los recursos computacionales periódicamente. Mención especial requiere la gestión de los recursos humanos, gran clave para el logro de los objetivos propuestos, donde las motivaciones y niveles de compromiso fueron inicialmente de alta heterogeneidad.

El respeto a los derechos de los pacientes fue siempre lo principal, determinando protocolos, diseñando flujos de como efectuar el reclamo y/o sugerencias, su trámite interno y externo, su respuesta, el manejo de los consentimientos informados, los reclamos efectuados en línea y finalmente la centralización en el comité que agrupa los reclamos y propone soluciones, más allá de la solución individual inmediata. La dinámica de la clínica exigió normativa de aseo y limpieza tres veces al día de las clínicas, el establecimiento de bitácora de los equipos desde los simples teclados de cada computador hasta los grandes compresores generales. Todas estas tareas significaron personas, protocolos y nuevos costos directos e indirectos que incrementaron los gastos operacionales, que permiten revisar los estándares periódicamente y establecen nuevas dinámicas que superen la calidad del servicio cada período(6).

Las razones y el proceso de desarrollo de las medidas de rendimiento y argumentos para que la facultad de odontología implemente procesos de medición de la calidad en sus programas clínicos, condujeron a la mejora de las prácticas clínicas en el presente y fue hecho en la certeza que preparará mejor a sus graduados para sus futuras prácticas.

Se observó debilidad en la aplicación del modelo biopsicosocial, requiriéndose profundizar e incluir mayores contenidos en los planes de estudios para la mejor comprensión y evaluación de los pacientes ${ }^{(23)}$. Adicionalmente se observó necesario realizar investigaciones y mejoras metodológicas en la educación odontológica que provean estrategias suplementarias y nuevas tecnologías para involucrar a los estudiantes y mejorar su satisfacción con el aprendizaje ${ }^{(24)}$.

Actualmente en Chile, como en otras partes del mundo, tanto la educación como la práctica de la odontología, son afectados por incremento de la presión asistencial y por asegurar o mejorar la calidad de los cuidados odontológicos, y se espera que la profesión, incluya mayor responsabilidad en la mantención y mejora de la calidad de los cuidados odontológicos ${ }^{(25)}$.

Serán tareas durante el próximo trienio, evaluar en el tiempo los objetivos alcanzados en el cumplimiento de los estándares aplicables al Centro, promover la clínica de mantención de los pacientes en todas sus áreas, profundizar en lo académico las medidas de calidad, manteniendo la revisión en terreno de documentos, entrevistas y constataciones oculares con pauta de cotejo, el uso del tiempo de los estudiantes y su relación con el stress. 


\section{CONCLUSIONES}

Lograr las competencias de planificación, gestión sanitaria y elevar los niveles de calidad en la atención de pacientes de un centro de salud que incluye mayoritariamente las prestaciones de una Facultad de Odontología, fue un desafío continuo en el tiempo que involucró a toda la comunidad, requirió especialistas del área de calidad, incrementó los costos de operación institucional y finalmente condujo a su acreditación como centro prestador institucional de atención abierta del Ministerio de Salud.

\section{CONFLICTOS DE INTERÉS}

Los autores declaran no presentar conflictos de interés

\section{AGRADECIMIENTOS}

Por ayuda técnica al Sr. Sergio Ayala Toro, Jefe Administrativo, Centro de Salud San Bernardo por su aporte de información relevante sobre los procesos administrativos.

\section{FINANCIAMIENTO}

Este trabajo no cuenta con financiamiento alguno.

\section{Bibliografía}

1. Cooley RA. Measuring the quality of care: the importance of dentist involvement in determining quality measures. Acad Gen Dent Impact. 2016;10:28-9.

2. Institute of Medicine. Best care at lower cost: the path to continuously learning health care in America. Washington DC : Natl. Acad. Press; 2013.

3. Breault MR. Dental Quality Alliance: a practitioner's perspective-empowering the profession to measure its performance for the betterment of oral health. N Y State Dent J. 2017;83:11-4.

4. Herndon JB, Crall JJ, Aravamudhan K, Catalanotto FA, Huang IC, Rudner N, et al. Developing and testing pediatric oral healthcare quality measures. J Public Health Dent. 2015;75:191-201.

5. Hunt RJ, Aravamudhan K. The quality movement in oral health care: who will lead? J Am Dent Assoc. 2014;145:421-3.

6. Hunt RJ, Ojha D. Oral health care quality measurement and its role in dental education. J Dent Educ. 2017;81:1395-404.

7. Ojha D, Aravamudhan K. Leading the dental quality movement: a dental quality alliance perspective. J Calif Dent Assoc. 2016;44:239-44.

8. National Quality Forum. Quality measurement 101. 2018;1-23. [Consultado 02/12/2019] Disponible en: https://aaos.org/globalassets/quality-and-practiceresources/quality-performance-measures-resouces/pm_methods_v2.pdf

9. Evans RW. The monitor practice program: implications for dentistry and dental education. Aust Dent J. 2019;64:193-8.

10. Van Assche N, Fickl S, Francisco H, Gurzawska K, Milinkovic I, Navarro JM, et al. Guidelines for development of implant dentistry in the next 10 years regarding innovation, education, certification, and associations. Clin Oral Implants Res. 2018;29:568-575

11. MINSAL, Decreto Fuerza de Ley $N^{\circ} 1$, 2005. [consultado 08/04/2020] Disponible en: http://www.supersalud.gob.cl/normativa/668/articles-2621 recurso 1.pdf

12. Reglamento del Sistema de Acreditación para los Prestadores Institucionales de Salud [consultado 08/04/2020] Disponible en: http://www.supersalud.gob.cl/ normativa/668/articles-4788 recurso 1.pdf

13. Manual del Estándar General de Acreditación para Prestadores Institucionales de Atención Abierta. [consultado 08/04/2020] Disponible en: https://www.minsal.cl/ portal/url/item/b02648d692c847c2e04001011e012223.pdf 20 de junio 2019.

14. Registro de Entidades Acreditadoras [consultado 08/04/2020] Disponible en: http://www.supersalud.gob.cl/acreditacion/673/w3-propertyvalue-4743.html.
15. Acreditación de Prestadores. Superintendencia de Salud. [consultado 08/04/2020] Disponible en: http://www.supersalud.gob.cl/consultas/667/w3article-6236.html.

16. Guía Práctica para el Proceso de Acreditación de Prestadores Institucionales de Salud. [consultado 08/04/2020] Disponible en: http://www.supersalud.gob.cl/ observatorio/671/articles-8981_guia_practica_acreditacion.pdf.

17. Observatorio de Calidad en Salud Guía Práctica [consultado 08/04/2020] Disponible en: http://www.supersalud.gob.cl/observatorio/671/articles-16609_ recurso_1.pdf

18. Pauta de cotejo, Manual de Atención Abierta. Superintendencia de Salud. [consultado 08/04/2020] Disponible en: http://www.supersalud.gob.cl/ observatorio/671/articles-4530 Pauta AA pdf.pdf).

19. Bueno J, Gascon J, Saturno P. La otra perspectiva. Aten Primaria. 2002;30:43334.

20. Riveros J, Berne C, Mugica JM. Gestión y satisfacción en servicios de salud de Chile: Contraste entre las percepciones de los funcionarios y los usuarios. Rev Med Chile. 2010;138:630-638.

21. Montero-Pérez FJ, Calderón de La Barca-Gázmez JM, Jimenez-Murillo L, Quero-Espinoza F de B, Gracia-Garcia F, Roig-Garcia JJ. Gestión clínica de un servicio de urgencias hospitalario mediante un cuadro de mando asistencial específico. Emergencias. 2012;24:476-84.

22. Groene O, Brandt E, Schmidt W, Moeller J. The balanced scorecard of acute settings: development process, definition of 20 strategic objectives and implementation. Int J Qual Health Care. 2009;21:259-71.

23. Häggman-Henrikson B, Ekberg EC, Ettlin DA, Michelotti A, Durham J, Goulet JP, et al. Mind the Gap: a systematic review of implementation of screening for psychological comorbidity in dental and dental hygiene education. J Dent Educ. 2018;82:1065-76.

24. Sipiyaruk K, Gallagher JE, Hatzipanagos S, Reynolds PA. A rapid review of serious games: from healthcare education to dental education. Eur J Dent Educ. 2018;22, 243-257

25. Yamalik N. Quality systems in dentistry part 2. Quality assurance and improvement (QA/I) tools that have implications for dentistry. Int Dent J. 2007;57:459-67. 